ORIGINAL ARTICLE

\title{
Hyperchloraemic metabolic acidosis following open cardiac surgery
}

\author{
M Hatherill, S Salie, Z Waggie, J Lawrenson, J Hewitson, L Reynolds, A Argent
}

Arch Dis Child 2005;90:1288-1292. doi: 10.1136/adc.2005.078006

See end of article for authors' affiliations

.....................

Correspondence to: Dr M Hatherill, South African Tuberculosis

Vaccine Initiative, IIDMM and School of Child \& Adolescent Health, University of Cape Town, Anzio Road, Observatory, Cape Town 7925, South Africa; mark@rmh.uct. ac.za

Accepted

1 September 2005

Published Online First

13 September 2005

\begin{abstract}
Aims: To describe acid-base derangements in children following open cardiac surgery on cardiopulmonary bypass (CPB), using the Fencl-Stewart strong ion approach.

Methods: Prospective observational study set in the paediatric intensive care unit (PICU) of a university children's hospital. Arterial blood gas parameters, serum electrolytes, strong ion difference, strong ion gap (SIG), and partitioned base excess (BE) were measured and calculated on admission to PICU.

Results: A total of 97 children, median age 57 months (range 0.03-166), median weight $14 \mathrm{~kg}$ (range 2.1-50), were studied. Median CPB time was 80 minutes (range 17-232). Predicted mortality was $2 \%$ and there was a single non-survivor. These children showed mild metabolic acidosis (median standard bicarbonate $20.1 \mathrm{mmol} / \mathrm{l}, \mathrm{BE}-5.1 \mathrm{mEq} / \mathrm{l}$ ) characterised by hyperchloraemia (median corrected $\mathrm{Cl}$ $113 \mathrm{mmol} / \mathrm{ll}$ ), and hypoalbuminaemia (median albumin $30 \mathrm{~g} / \mathrm{l}$ ), but no significant excess unmeasured anions or cations (median SIG $0.7 \mathrm{mEq} / \mathrm{l}$ ). The major determinants of the net $\mathrm{BE}$ were the chloride and albumin components (chloride effect $-4.8 \mathrm{mEq} / \mathrm{l}$, albumin effect $+3.4 \mathrm{mEq} / \mathrm{l}$ ). Metabolic acidosis occurred in 72 children (74\%) but was not associated with increased morbidity. Hyperchloraemia was a causative factor in 53 children $(74 \%)$ with metabolic acidosis. Three $(4 \%)$ hyperchloraemic children required adrenaline for inotropic support, compared to eight children $(28 \%)$ without hyperchloraemia. Hypoalbuminaemia was associated with longer duration of inotropic support and PICU stay.

Conclusions: In these children with low mortality following open cardiac surgery, hypoalbuminaemia and hyperchloraemia were the predominant acid-base abnormalities. Hyperchloraemia was associated with reduced requirement for adrenaline therapy. It is suggested that hyperchloraemic metabolic acidosis is a benign phenomenon that should not prompt escalation of haemodynamic support. By contrast, hypoalbuminaemia, an alkalinising force, was associated with prolonged requirement for intensive care.
\end{abstract}

$\mathrm{T}$ e presence of metabolic acidosis in a child following cardiac surgery might prompt the clinician to escalate haemodynamic support, based on the assumption that metabolic acidosis signifies low cardiac output, poor tissue perfusion, and increased risk of adverse events. ${ }^{1-5}$ There are pitfalls in this approach, since outcome in critically ill children may depend on the underlying mechanism (lactate, chloride, unmeasured anions), rather than the magnitude of the acidosis. ${ }^{6}$

Hyperlactataemia following cardiac surgery has been associated with postoperative adverse events and mortality. ${ }^{478}$ However, mild lactic acidosis following cardiac surgery on cardiopulmonary bypass (CPB) does not necessarily signify regional tissue hypoxia or intraoperative oxygen debt, since it may be influenced by confounding factors such as glycolytic flux, or pyruvate dehydrogenase inhibition. ${ }^{9-11}$ Postoperative metabolic acidosis may also be influenced by the choice of fluid used to prime the $\mathrm{CPB}$ circuit, contributing to hyperlactataemia, hyperchloraemia, or increase of unmeasured anions. ${ }^{12-15}$ However, in contrast to hyperlactataemia, the clinical significance of hyperchloraemic acidosis following cardiac surgery with CPB is unclear. ${ }^{4578}$

We speculated that metabolic acidosis following open cardiac surgery in children is more often due to hyperchloraemia, rather than to hyperlactataemia, and secondly, that hyperchloraemic metabolic acidosis is a benign phenomenon that should not prompt escalation of cardiac support in the immediate postoperative period. This study attempts to delineate the mechanism and clinical significance of acidbase derangements in children following open cardiac surgery, in terms of a modified Fencl-Stewart strong ion approach. ${ }^{16-21}$

\section{METHODS}

The study was set in the paediatric intensive care unit (PICU) of a university children's hospital in Cape Town, South Africa. This regional PICU is staffed for 18-22 beds, admits approximately 1200 children per year, and has full time paediatric intensivist cover. The children's hospital functions as the paediatric cardiology and cardiothoracic surgical referral centre for the Western Cape Province, which has a population of approximately 4 million people. Between 250 and 300 paediatric cardiac operations are performed per annum, of which approximately 170 are open cases using CPB. Neither surgical palliation of hypoplastic left heart syndrome (Norwood procedure), nor extracorporeal membrane oxygenation (ECMO), are currently offered at this centre.

All children admitted to the PICU following CPB for surgical correction of congenital or acquired heart defects during the calendar year 2003 were screened for enrolment, during the duty periods of the principal investigator $(\mathrm{MH})$. Cardiopulmonary bypass was the only criterion for eligibility-that is, children were not selected on the basis of anatomical defect or type of surgery. The fluid used to prime the CPB circuit (the "pump prime") was usually a mixture of blood and stabilised human serum (SHS), a colloid prepared by the regional blood transfusion service (Western Province Blood Transfusion Service, Parow, South Africa). In children with severe preoperative polycythaemia, the pump prime

Abbreviations: $A B G$, arterial blood gas; $B E$, base excess; $C P B$, cardiopulmonary bypass; ECMO, extracorporeal membrane oxygenation; PICU, paediatric intensive care unit; SHS, stabilised human serum; SID, strong ion difference; SIG, strong ion gap 
consisted of SHS alone. SHS contains $36 \mathrm{~g} / \mathrm{l}$ albumin, $13 \mathrm{~g} / \mathrm{l}$ immunoglobulin, $130 \mathrm{mmol} / \mathrm{l}$ sodium, and $130 \mathrm{mmol} / \mathrm{l}$ chloride. Blood and SHS were also the fluids of choice for intraoperative volume resuscitation, rather than $0.9 \%$ saline or Ringer's lactate.

Immediately on admission to PICU, blood was routinely sampled for arterial blood gas (ABG) analysis and electrolytes. Blood $\mathrm{pH}, \mathrm{pCO}_{2}$, bicarbonate, and standard base excess (BE) were measured and derived using a Radiometer ABL 520 blood gas analyser (Copenhagen, Denmark). Serum electrolytes (sodium, potassium, calcium, magnesium, phosphate) were measured by the ion specific electrode method using a Beckman CX9 Pro analyser (Berlin, Germany). Serum lactate was measured by the enzymatic method using a Beckman CX5 analyser (Berlin, Germany). Serum albumin was measured by the reagent method using a Beckman CX9 Pro analyser (Berlin, Germany). Metabolic acidosis was defined as standard bicarbonate (SB) $<22 \mathrm{mmol} / \mathrm{l}$. Clinically significant biochemical derangements were defined as albumin $<30 \mathrm{~g} / \mathrm{l}$, chloride $>110 \mathrm{mmol} / \mathrm{l}$, lactate $>2 \mathrm{mmol} / \mathrm{l}$, and SIG $>2 \mathrm{mmol} / \mathrm{l}$ ( see table 1 ).

A Fencl-Stewart approach, with the modifications of Figge, was used to derive calculated strong ion difference (SIDc), effective strong ion difference (SIDe), and strong ion gap (SIG). ${ }^{16-20}$ Chloride was corrected for free water $(\mathrm{cCl})$ - that is, to a serum sodium of $140 \mathrm{mmol} / \mathrm{l}$, by multiplying the measured chloride:sodium ratio by a factor of 140 (see table 1).

The individual components of the standard base excess (BE) contributed by albumin (BE alb), free water (BE fw), chloride (BE cl), and lactate (BE lact), were calculated using the equations of Gilfix, incorporating normal values for the ion sensitive electrode method. ${ }^{21-23}$ For the purposes of these calculations, reference values were taken as albumin $42 \mathrm{~g} / \mathrm{l}$, sodium $140 \mathrm{mmol} / \mathrm{l}$, chloride $108 \mathrm{mmol} / \mathrm{l}$, and lactate $1.5 \mathrm{mmol} / \mathrm{l}^{22}{ }^{23}$ (see table 2 ).

Cardiac diagnoses and surgical procedures, duration of $\mathrm{CPB}$, duration of aortic cross-clamp, \% predicted mortality (using Paediatric Index of Mortality 1 at the time of the study period), duration of ventilation, duration of inotropic support, and duration of PICU stay (expressed as calendar days, or part thereof), and observed PICU mortality, were recorded. ${ }^{24}$

Ethics approval was obtained from the university ethics committee. Continuous and categorical data were analysed by the Mann-Whitney and Fisher's exact tests respectively, and by linear regression, using Analyse-It statistical software (Analyse-It, UK). Data are reported as median (range), and $\mathrm{n}$ (\%).

Two hundred and seventy eight paediatric cardiac surgical procedures were performed during the study period. One hundred and sixty nine $(61 \%)$ were open procedures on CPB, of which 97 children (57\%), median age 57 months (range 0.03-166), median weight $14 \mathrm{~kg}$ (range 2.1-50), were studied.

Table 1 Equations and definitions

\begin{tabular}{ll}
\hline & Definition \\
\hline Metabolic acidosis & $\mathrm{SB}<22 \mathrm{mmol} / \mathrm{I}$ \\
Corrected chloride (cCl) & $140 \times \mathrm{Cl} / \mathrm{Na}$ \\
Hyperchloraemia & $\mathrm{Cl}>110 \mathrm{mmol} / \mathrm{I}$ \\
Raised lactate & $>2 \mathrm{mmol} / \mathrm{I}$ \\
Raised SIG & $>2 \mathrm{mmol} / /$ \\
Low albumin & $<30 \mathrm{~g} / \mathrm{I}$ \\
SIDc & $\mathrm{Na}+\mathrm{K}+\mathrm{Ca}+\mathrm{Mg}-(\mathrm{Cl}+$ lactate) \\
SIDe & Bicarbonate $+\mathrm{PO}_{4}$ charge + albumin \\
& charge \\
Strong ion gap (SIG) & SIDc $-\mathrm{SIDe}$ \\
\hline
\end{tabular}

Table 2 Equations and definitions: calculated components of total base excess (BE)

\begin{tabular}{lll}
\hline & & Definition \\
\hline Albumin & {$[\mathrm{BE}(\mathrm{alb})]$} & $(0.123 \times \mathrm{pH}-0.631) \times(42-$ albumin $)$ \\
Free water & {$[\mathrm{BE}(\mathrm{fw})]$} & $0.3 \times(\mathrm{Na}-140)$ \\
Chloride & {$[\mathrm{BE}(\mathrm{cl})]$} & $108-\mathrm{Cl}$ \\
Lactate & {$[\mathrm{BE}($ lact $)]$} & $1.5-$ lactate \\
\hline
\end{tabular}

\section{RESULTS}

During the study period, paediatric cardiac surgical mortality was $3.6 \%(n=10 / 278)$ overall, and $2.4 \%(n=4 / 169)$ for open cases on CPB. Median predicted mortality was $2 \%$ (range $1-59 \%)$ for the study group $(\mathrm{n}=97)$, in which there was a single non-survivor (observed mortality 1\%). All 97 children were mechanically ventilated, with median duration of ventilation of 2 calendar days (range 1-16). Ninety five children (98\%) were receiving inotropic support on return from theatre, of whom $11(11 \%)$ received adrenaline by continuous infusion. Median duration of inotropic support was 3 calendar days (range $0-10$ ). Ninety two children (95\%) had two organ system failures (all cardiac and respiratory) and five children $(5 \%)$ had three or more organ system failures. Median duration of PICU stay was 4 calendar days (range 2-20).

Cardiac surgical diagnoses, stratified by Risk Adjustment in Congenital Heart Surgery (RACHS-1) categories, are shown in table $3 .{ }^{25}$ Median CPB time was 80 minutes (range 17-232) and median aortic cross-clamp time was 46 minutes (range 0-149). Aortic cross-clamp time had weak, but statistically significant, relationships with the variation in admission lactate (slope $+0.008 ; \mathrm{R}^{2} 0.03 ; \mathrm{p}<0.0001$ ); $\mathrm{cCl}$ (slope +0.027; $\mathrm{R}^{2} 0.04 ; \mathrm{p}<0.0001$ ); SIG (slope -0.020 ; $\mathrm{R}^{2} 0.02 ; \mathrm{p}=0.05$ ); and albumin (slope $-0.016 ; \mathrm{R}^{2} 0.01$; $\mathrm{p}<0.0001)$.

Table 3 Cardiac surgical procedures stratified by RACHS-1 risk categories ${ }^{25}$

\begin{tabular}{|c|c|c|}
\hline Risk category & No. & $(\%)$ \\
\hline $\begin{array}{l}\text { Risk category } 1 \\
\text { Atrial septal defect (ASD) repair } \\
\text { Risk category } 2 \\
\text { Aortic valve repair } \\
\text { Total anomalous pulmonary venous } \\
\text { drainage (TAPVD) repair } \\
\text { Pulmonary stenosis repair } \\
\text { Glenn shunt } \\
\text { Tetralogy of Fallot repair } \\
\text { Ventricular septal defect (VSD) repair } \\
\text { Risk category } 3 \\
\text { Mustard procedure } \\
\text { Takeuchi procedure } \\
\text { Arterial switch procedure } \\
\text { Excision of intracardiac tumour } \\
\text { Ross procedure } \\
\text { Right ventricular outflow tract augmentation } \\
\text { Right ventricular pulmonary artery conduit } \\
\text { Atrioventricular canal defect (AVSD) repair } \\
\text { Total cavo-pulmonary connection (TCPC) } \\
\text { Mitral valve repair or replacement } \\
\text { Risk category } 4 \\
\text { Repair of truncus arteriosus } \\
\text { Aortic arch repair } \\
\text { Risk category } 5 \\
\text { Nil } \\
\text { Risk category } 6 \\
\text { Damus-Kaye-Stansel procedure }\end{array}$ & $\begin{array}{l}(n=3 \\
(n=3) \\
(n=46 \\
(n=2) \\
(n=3) \\
(n=4) \\
(n=7) \\
(n=) \\
(n=9) \\
(n=21) \\
(n=44 \\
(n=1) \\
(n=1) \\
(n=1) \\
(n=1) \\
(n=3) \\
(n=3) \\
(n=5) \\
(n=7) \\
(n=8) \\
(n=14) \\
(n=3) \\
(n=2) \\
(n=1)\end{array}$ & $\begin{array}{l}(3 \%) \\
(47 \%)\end{array}$ \\
\hline
\end{tabular}




\begin{tabular}{|c|c|c|}
\hline Parameter & Median & (range) \\
\hline $\mathrm{pH}$ & 7.38 & (7.17-7.61) \\
\hline $\mathrm{BE}(\mathrm{mEq} / \mathrm{l})$ & -5.1 & $(-12.9$ to +2.5$)$ \\
\hline $\mathrm{SB}(\mathrm{mmol} / \mathrm{l})$ & 20.1 & $(10.6-28.8)$ \\
\hline Albumin (g/l) & 30 & $(16-44)$ \\
\hline Lactate $(\mathrm{mmol} / \mathrm{l})$ & 1.8 & $(0.7-9.1)$ \\
\hline Sodium $(\mathrm{mmol} / \mathrm{l})$ & 138 & $(129-146)$ \\
\hline Chloride (mmol/l) & 111 & $(97-121)$ \\
\hline $\mathrm{cCl}(\mathrm{mmol} / \mathrm{l})$ & 113 & $(101-126)$ \\
\hline $\mathrm{SIDc}(\mathrm{mEq} / \mathrm{l})$ & 31.7 & $(19.7-46.5)$ \\
\hline $\mathrm{SIDe}(\mathrm{mEq} / \mathrm{l})$ & 31.1 & $(22.9-40.9)$ \\
\hline Strong ion gap $(\mathrm{mEq} / \mathrm{l})$ & 0.7 & $(-13.7$ to +14.8 \\
\hline
\end{tabular}

Acid-base data immediately on admission to PICU after cardiac surgery are shown in table 4 . This group of children showed mild metabolic acidosis (median SB $20.1 \mathrm{mmol} / \mathrm{l}$ and $\mathrm{BE}-5.1 \mathrm{mEq} / \mathrm{l}$ ) characterised by hyperchloraemia (median $\mathrm{cCl} 113 \mathrm{mmol} / \mathrm{l})$. Half of the patients showed clinically significant hypoalbuminaemia (median albumin $30 \mathrm{~g} / \mathrm{l}$ ). There was no clinically significant excess of "truly unmeasured" anions or cations (median SIG $0.7 \mathrm{mEq} / \mathrm{l}$ ).

\section{Partitioned base excess (BE)}

Calculated components of the total base excess are shown in table 5. The primary individual determinants of the total BE were chloride and albumin. The predominant contribution to the negative total base excess (median $\mathrm{BE}-5.1 \mathrm{mEq} / \mathrm{l}$ ) was the chloride component (median $\mathrm{BE} \mathrm{cl}-4.8 \mathrm{mEq} / \mathrm{l}$ ), with a minor contribution by lactate and free water (median BE lact $-0.3 \mathrm{mEq} / \mathrm{l}$ and $\mathrm{BE} \mathrm{fW}-0.6 \mathrm{mEq} / \mathrm{l})$. The negative $\mathrm{BE}$ contributions were partly offset by the positive albumin component (median BE alb $+3.4 \mathrm{mEq} / \mathrm{l}$ ). The median $\mathrm{BE}$ component due to the net effect of other measured (calcium, magnesium, phosphate) and "truly unmeasured" cations and anions was $-1.6 \mathrm{mEq} / \mathrm{l}$.

\section{Albumin}

Forty six children had clinically significant hypoalbuminaemia (47\%). Hypoalbuminaemia was associated with a longer duration of inotropic support, median 3 days (range 1-10) compared to 2 days (range $0-10)(p=0.047)$, and longer duration of PICU stay, median 4 days (range 2-11) compared to 3 days (range $2-20)(p=0.009)$. There was no significant association between hypoalbuminaemia and increased adrenaline requirement $(p=0.14), C P B$ time $(p=0.55)$, aortic cross-clamp time $(p=0.29)$, predicted mortality $(p=0.17)$, or duration of mechanical ventilation $(\mathrm{p}=0.09)$.

\section{Metabolic acidosis}

Seventy two children (74\%) had a metabolic acidosis (see table 6). The presence of metabolic acidosis was not associated with increased adrenaline requirement $(p=0.69), C P B$ time $(p=0.52)$, aortic cross-clamp time $(\mathrm{p}=0.61)$, predicted mortality $(\mathrm{p}=0.35)$, duration of mechanical ventilation $(p=0.99)$, duration of inotropic support $(p=0.53)$, or duration of PICU stay $(p=0.65)$.

Table 5 Calculated components of total base excess

\begin{tabular}{lll}
\hline Parameter & Median & (range) \\
\hline BE (alb) & +3.4 & $(-0.6$ to +6.8$)$ \\
BE (fw) & -0.6 & $(-3.3$ to +1.8$)$ \\
BE (cl) & -4.8 & $(-18$ to +7.2$)$ \\
BE (lact) & -0.3 & $(-7.6$ to +0.8$)$ \\
\hline
\end{tabular}

Table 6 Incidence of individual metabolic acid-base abnormalities

\begin{tabular}{lll}
\hline Abnormality & No. & $(\%)$ \\
\hline Low albumin & $n=46$ & $(47 \%)$ \\
Hyperchloraemia & $n=68$ & $(70 \%)$ \\
Hyperlactataemia & $n=41$ & $(42 \%)$ \\
Raised SIG & $n=39$ & $(40 \%)$ \\
Metabolic acidosis & $n=72$ & $(74 \%)$ \\
Single primary aetiology & $n=34$ & $(47 \%)$ \\
$\quad$ Hyperchloraemia & $n=24$ & $(33 \%)$ \\
Lactic & $n=1$ & $(1 \%)$ \\
Raised SIG & $n=9$ & $(13 \%)$ \\
Mixed aetiology & $n=38$ & $(53 \%)$ \\
$\quad$ Hyperchloraemia & $n=29$ & $(40 \%)$ \\
Other & $n=9$ & $(13 \%)$ \\
\hline
\end{tabular}

There was a single primary cause for the metabolic acidosis $(n=72)$ in 34 children $(47 \%)$. The primary cause of metabolic acidosis was hyperchloraemia in 24 children (33\%). Other primary causes of metabolic acidosis were rare, with raised lactate in one child $(1 \%)$, and raised SIG in nine children (13\%). The cause of the metabolic acidosis was mixed in 38 children (53\%), in whom hyperchloraemia was one of the causative factors in 29 children (40\%). In total, hyperchloraemia was a causative factor in 53 children (74\%) with metabolic acidosis.

\section{Chloride}

Hyperchloraemia was associated with reduced adrenaline requirement, with three $(4 \%)$ hyperchloraemic children receiving adrenaline, compared to eight $(28 \%)$ without hyperchloraemia $(p=0.005)$. However, there was no association between hyperchloraemia and increased CPB time $(\mathrm{p}=0.31)$, aortic cross-clamp time $(\mathrm{p}=0.68)$, predicted mortality $(p=0.21)$, duration of inotrope requirement $(p=0.90)$, mechanical ventilation $(p=0.29)$, or PICU stay $(\mathrm{p}=0.17)$.

\section{DISCUSSION}

The associations between cardiac surgical mortality, serious adverse events, and lactic acidosis in the immediate postoperative period have been well documented. ${ }^{4} 58$ However, these studies reported mortality rates of $4-17 \%$, whereas paediatric cardiac surgical mortality has fallen to $<2 \%$ in some centres. ${ }^{457826}$ The lactic acidosis outcome model may need to be reviewed in light of better postoperative survival, driven partly by general improvements in the outcome of paediatric intensive care and goal directed treatment guidelines. ${ }^{26} 27$

This study has shown that although metabolic acidosis is common following CPB, it is rarely due to increase of lactate. Murray and colleagues have shown, in a mixed group of 44 children following both open and closed cardiac surgery, that metabolic acidosis due to hyperlactataemia was rare, whereas increase of "unmeasured" anions was the most common cause of metabolic acidosis in their study population. ${ }^{28}$ In a sub-group of children undergoing CPB, hyperchloraemia was common, although the clinical significance of this abnormality was not clear. ${ }^{28}$ Durward et al also showed, in children following $\mathrm{CPB}$, that metabolic acidosis was most commonly due to either increase of the SIG or hyperchloraemia. ${ }^{29}$

The prognostic significance of acid-base data is difficult to evaluate when cardiac surgical mortality is $<2 \%$. We have used parameters such as predicted mortality and duration of intensive care as surrogate adverse endpoints. Despite the frequency of metabolic acidosis in this study, metabolic acidosis was not associated with severity of insult (CPB or 


\section{What is already known on this topic}

- Raised lactate after cardiac surgery is associated with postoperative adverse events and mortality

- Metabolic acidosis may be influenced by the type of fluid used to prime the cardiopulmonary bypass circuit

aortic cross-clamp time), risk of death, or longer duration of cardio-respiratory support in the PICU.

Hypoalbuminaemia is associated with both prolonged ICU stay and mortality in critical illness. ${ }^{30}{ }^{31}$ We have shown that hypoalbuminaemia is common in children following open cardiac surgery. Since preoperative serum albumin data were not collected, it is not possible to determine whether this finding is a consequence of nutrition, haemodilution, or an intraoperative acute phase response. Hypoalbuminaemia contributed a substantial positive component to the total base excess, an effect which might lead the clinician to underestimate the magnitude of an underlying metabolic acidosis. Given that hypoalbuminaemia, an alkalinising force, was associated with longer duration of both inotropic support and PICU stay, it is unsurprising that metabolic acidosis per se was not associated with these adverse endpoints. ${ }^{32} 33$

Hyperchloraemia was the most common cause of metabolic acidosis in these children and contributed a substantial negative component to the total base excess. Animal work has shown that saline resuscitation leading to hyperchloraemic acidosis is less effective than resuscitation with a balanced electrolyte colloid solution, but it is not clear whether these findings may be extrapolated to intrinsic, rather than extrinsic, hyperchloraemic acidosis. ${ }^{34}$ Our hypothesis that postoperative hyperchloraemic metabolic acidosis is a benign phenomenon that might not require escalation of therapy is supported by the data, in that hyperchloraemia was not associated with longer CPB, aortic cross-clamp time, or duration of cardio-respiratory support. Moreover, children with hyperchloraemia were less likely to require adrenaline infusion for inotropic support on return from the operating theatre.

The origin of the excess chloride may be the fluid used to prime the CPB circuit. Although the pump prime did not contain $0.9 \%$ saline, the colloid preparation contained chloride and sodium in a similar $1: 1$ ratio $(130 \mathrm{mmol} / \mathrm{l})$, which would tend to narrow the strong ion difference and generate hyperchloraemic metabolic acidosis. ${ }^{15}{ }^{35}$ It is also possible that renal perfusion is impaired during $\mathrm{CPB}$, even in the absence of regional tissue hypoxia, leading to acute tubular necrosis with chloride sparing natriuresis. ${ }^{1036}$

Although it is generally accepted that sodium bicarbonate therapy is not appropriate for lactic acidosis, a case might be made for sodium replacement in hyperchloraemic acidosis, using sodium bicarbonate to lower the chloride:sodium ratio and increase the strong ion difference, rather than to replace bicarbonate. ${ }^{37-39}$ In a haemodynamically stable postoperative patient, both renal impairment and the associated hyperchloraemic acidosis might be expected to resolve spontaneously, without escalation of cardiac support. In this study, the lack of association between hyperchloraemia and adverse endpoints, and the inverse association with adrenaline use, supports the view that hyperchloraemic metabolic acidosis does not require active intervention. Whether these findings might be extrapolated to postoperative metabolic acidosis after other types of surgery is a matter for further investigation.

In contrast to the findings of Murray et al, we found no excess "truly unmeasured" anions reflected in the SIG of this
What this study adds

- Hyperchloraemic metabolic acidosis is common after open cardiac surgery and may be a benign phenomenon that does not require escalation of haemodynamic support

- Hypoalbuminaemia, an alkalinising force, is associated with prolonged intensive care

patient population. ${ }^{28}$ This finding may be due to a real absence of such anions, or the simultaneous presence of excess unmeasured cations. Therefore the differences between our findings and those of Murray might be ascribed to the fact that all of our patients underwent cardiopulmonary bypass. ${ }^{15}{ }^{28}$ We speculated that such unmeasured cations might be derived from the globulin containing blood product used to prime the CPB circuit. $^{40}{ }^{41}$ However, Durward et al showed not only that the SIG was a common cause of metabolic acidosis after CPB, but that the SIG was superior to lactate as a predictor of mortality following open cardiac surgery. ${ }^{29}$ While there may have been differences in case mix between that patient population and our own, it is interesting to note that hyperchloraemia was also associated with survival in that study. ${ }^{29}$

Mortality in this study group was only $1 \%$. Patients were not pre-selected by diagnosis or surgical procedure at the time of enrolment and we believe the study group is representative of children undergoing open cardiac surgery at this institution, for whom overall mortality was comparable at $2.4 \%$. However, palliative surgery for hypoplastic left heart syndrome is not offered at this centre and more than $90 \%$ of patients fell into surgical risk categories 2 and $3 .{ }^{25}$ Therefore, our patient group may have a different morbidity profile, and spectrum of acid-base derangement, from that of centres in which high risk procedures form a larger proportion of the paediatric cardiac surgical workload.

\section{Conclusion}

In this group of children with low mortality following open cardiac surgery, hypoalbuminaemia and hyperchloraemia were the predominant acid-base abnormalities, whereas lactic acidosis, and SIG acidosis were rare. Hyperchloraemia following cardiopulmonary bypass appears to be a benign phenomenon and we suggest that hyperchloraemic metabolic acidosis should not prompt escalation of haemodynamic support. By contrast, hypoalbuminaemia, an alkalinising force, was associated with prolonged requirement for intensive care.

\section{Authors' affiliations}

M Hatherill, S Salie, Z Waggie, J Lawrenson, J Hewitson, L Reynolds, A Argent, Division of Critical Care \& Children's Heart Disease, School of Child \& Adolescent Health, University of Cape Town, South Africa

Competing interests: none

\section{REFERENCES}

1 Hoffman TM, Wernovsky G, Atz AM, et al. Efficacy and safety of milrinone in preventing low cardiac output syndrome in infants and children after corrective surgery for congenital heart disease. Circulation 2003; 107:996-1002.

2 Davis JW, Shackford SR, Holbrook TL. Base deficit as a sensitive indicator of compensated shock and tissue oxygen utilization. Surg Gynecol Obstet $1991 ; 173: 473-6$

3 Smith I, Kumar P, Molloy S, et al. Base excess and lactate as prognostic indicators for patients admitted to intensive care. Intensive Care Med 2001;27:74-83. 
4 Hatherill M, Saijanhar T, Tibby SM, et al. Serum lactate as a predictor of mortality after paediatric cardiac surgery. Arch Dis Child 1997;77:235-8

5 Duke T, Butt W, South $M$, et al. Early markers of major adverse events in children after cardiac operations. J Thorac Cardiovasc Surg 1997; 114:1042-52.

6 Hatherill M, Waggie Z, Purves L, et al. Mortality and the nature of metabolic acidosis in children with shock. Intensive Care Med 2003;29:286-91.

7 Cheifetz IM, Kern FH, Schulman SR, et al. Serum lactates correlate with mortality after operations for complex congenital heart disease. Ann Thorac Surg 1997;64:735-8

8 Siegel LB, Dalton HJ, Hertzog JH, et al. Initial postoperative serum lactate levels predict survival in children after open heart surgery. Intensive Care Med 1996;22:1418-23.

9 James JH, Luchette FA, McCarter FD, et al. Lactate is an unreliable indicator of tissue hypoxia in injury or sepsis. Lancet 1999;354:505-8.

10 Ganuschak YM, Maessen JG, De Jong DS. The oxygen debt during routine cardiac surgery: illusion or reality? Perfusion 2002; 17:167-73.

11 Li J, Schulze-Neick I, Lincoln C, et al. Oxygen consumption after cardiopulmonary bypass surgery in children: determinants and implications. J Thorac Cardiovasc Surg 2000;1 19:523-33.

12 Morgan TJ, Venkatesh B, Hall J. Crystalloid strong ion difference determine metabolic acid-base change during in vitro hemodilution. Crit Care Med 2002;30:157-60.

13 Sandstrom K, Nilsson K, Andreasson S, et al. Open heart surgery, pump prime effects and arteriovenous differences in glucose, lactate and ketones. Paediatr Anaesth 1999;9:53-9.

14 Himpe D, Neels H, De Hert S, et al. Adding lactate to the prime solution during hypothermic cardiopulmonary bypass: a quantitative acid-base analysis. Br J Anaesth 2003:90:440-5.

15 Liskaser FJ, Bellomo R, Hayhoe M, et al. Role of pump prime in the etiology and pathogenesis of cardiopulmonary bypass-associated acidosis. Anesthesiology 2000;93:1 170-3.

16 Stewart PA. Modern quantitative acid-base chemistry. Can J Physiol Pharmacol 1983;61:1444-61.

17 Fencl V, Jabor A, Kazda A, et al. Diagnosis of metabolic acid-base disturbances in critically ill patients. Am J Respir Crit Care Med 2000; 162:2246-51.

18 Figge $\mathrm{J}$, Rossing $\mathrm{TH}$, Fend $\mathrm{V}$. The role of serum proteins in acid-base equilibria. J Lab Clin Med 1991;117:453-67.

19 Figge J, Mydosh T, Fend V. Serum proteins and acid-base equilibria: a follow-up. J Lab Clin Med 1992; 120:713-19.

20 Kellum JA, Kramer DJ, Pinsky MR. Strong ion gap: a methodology for exploring unexplained anions. J Crit Care 1995; 10:51-5.

21 Gilfix BM, Bique M, Magder S. A physical chemical approach to the analysis of acid-base balance in the clinical setting. J Crit Care 1993;8:187-97.

22 Rose BD, Post TW. Metabolic acidosis. In Clinical physiology of acid-base and electrolyte disorders, 5th edn. New York, McGraw Hill.

23 Winter SD, Pearson R, Gabow PA, et al. The fall of the serum anion gap. Arch Intern Med 1990;150:311-13
24 Shann F, Pearson G, Slater A, et al. Paediatric index of mortality (PIM): a mortality prediction model for children in intensive care. Intensive Care Med 1997;23:201-7.

25 Jenkins KJ, Gauvreau K. Center-specific differences in mortality: preliminary analyses using the Risk Adjustment in Congenital Heart Surgery (RACHS-1) method. J Thorac Cardiovasc Surg 2002;124:97-104.

26 Rossi AF, Khan DM, Hannan R, et al. Goal-directed medical therapy and point-of-care testing improve outcomes after congenital heart surgery. Intensive Care Med 2005;31:98-104.

27 Frey B, Macrae DJ. Goal-directed therapy may improve outcome in complex patients - depending on the chosen treatment end point. Intensive Care Med 2005;31:508-9.

28 Murray DM, Olhsson V, Fraser Jl. Defining acidosis in postoperative cardiac patients using Stewart's method of strong ion difference. Pediatr Crit Care Med 2004;5:240-5.

29 Durward A, Tibby SM, Skellett S, et al. The strong ion gap predicts mortality in children following cardiopulmonary bypass surgery. Pediatr Crit Care Med 2005;6:281-5.

30 Durward A, Mayer A, Skellett S, et al. Hypoalbuminaemia in critically ill children: incidence, prognosis, and influence on the anion gap. Arch Dis Child 2003;88:419-22.

31 Blunt MC, Nicholson JP, Park GR. Serum albumin and colloid osmotic pressure in survivors and nonsurvivors of prolonged critical illness. Anaesthesia 1998:53:755-61.

32 McAuliffe JJ, Lind $\mathrm{U}$, Leith DE, et al. Hypoproteinemic alkalosis. Am J Med 1986;81:86-90.

33 Wilkes P. Hypoproteinemia, strong-ion difference, and acid-base status in critically ill patients. J Appl Physio 1998;84:1740-8.

34 Kellum JA. Fluid resuscitation and hyperchloraemic acidosis in experimental sepsis: improved short-term survival and acid-base balance with hextend compared with saline. Crit Care Med 2002:30:300-5.

35 Skellett S, Mayer A, Durward A, et al. Chasing the base deficit: hyperchloraemic acidosis following $0.9 \%$ saline fluid resuscitation. Arch Dis Child 2000;83:514-16.

36 Rose BD, Post TW. Meaning and application of urine chemistries. In Clinical physiology of acid-base and electrolyte disorders, 5th edn. New York, McGraw Hill.

37 Forsythe SM, Schmidt GA. Sodium bicarbonate for the treatment of lactic acidosis. Chest 2000;117:260-7.

38 Durward A, Skellett S, Mayer A, et al. The value of the chloride:sodium ratio in differentiating the aetiology of metabolic acidosis. Intensive Care Med 2001;27:828-35.

39 Rehm M, Finsterer U. Treating intraoperative hyperchloraemic acidosis with sodium bicarbonate or tris-hydroxymethyl aminomethane: a randomized prospective study. Anesth Analg 2003;96:1201-8.

40 De Troyer A, Stolarczyk A, De Beyl DZ, et al. Value of anion-gap determination in multiple myeloma. N Engl J Med 1977;296:858-60.

41 Nanji AA, Campbell DJ, Pudek MR. Decreased anion gap associated with hypoalbuminemia and polyclonal gammopathy. JAMA 1981;246:859-60. 